\title{
Tratamento do hemangioma de coróide e descolamento total da retina com vitrectomia posterior: relato de caso
}

\author{
Choroidal hemangioma with extensive retinaldetachment treated with posterior \\ vitrectomy:case report
}

Luciana Duarte Rodrigiues ${ }^{1}$ Luciana Lucci Serracarbassa ${ }^{2}$

Yoshitaka Nakashima ${ }^{3}$

Pedro Durães Serracarbassa ${ }^{4}$

\section{RESUMO}

Apresentar a evolução de um caso de hemangioma circunscrito da coróide associado a descolamento total da retina tratado com vitrectomia posterior e endofotocoagulação. Relato de caso intervencional. Paciente do sexo feminino, com 41 anos, apresentava mancha escura na visão do olho direito há uma semana, com progressão da mancha para o campo inferior e diminuição súbita da acuidade visual há dois dias. Ao exame oftalmológico, apresentava acuidade visual menor que 20/400 no olho direito. À fundoscopia do olho direito, observava-se elevação da retina neurossensorial nos quatro quadrantes e uma lesão sub-retiniana avermelhada no pólo posterior, com bordas pouco nítidas. O ultra-som do olho direito mostrava membranas móveis de alta refletividade na cavidade vítrea (retina) aderidas ao nervo óptico e presença de lesão sólida hiperecogênica, homogênea, no pólo posterior, com picos de média refletividade no seu interior, sugestiva de hemangioma de coróide Todos os exames sistêmicos foram normais. Optou-se pela vitrectomia posterior com endodrenagem, retinopexia, endofotocoagulação do tumor e colocação de gás $\mathrm{C}_{3} \mathrm{~F}_{8}$. A retina manteve-se aplicada nos quinze primeiros dias da cirurgia, quando então apresentou novo descolamento inferior da retina sobre o tumor. Optou-se por nova vitrectomia posterior com endofotocoagulação e colocação de óleo de silicone. A paciente encontra-se no $6^{\circ}$ mês de pós-operatório com a retina aplicada, óleo de silicone na cavidade vítrea, hemangioma com marcas de fotocoagulação (inativo?), acuidade visual igual a 20/400 no olho direito. A vitrectomia posterior surge como opção no tratamento do hemangioma da coróide associado a descolamentos extensos da retina, visando restabelecer a anatomia e diminuir as complicações funcionais tardias na retina.

Descritores: Hemangioma; Neoplasias da coróide; Vitrectomia; Descolamento retiniano; Relatos de casos [tipo de publicação]

\section{INTRODUÇ̃̃̃O}

O hemangioma da coróide é um hamartoma benigno relativamente raro, que pode se apresentar sob duas formas distintas: circunscrito e difuso ${ }^{(1)}$. A forma difusa está associada à angiomatose encefalo-facial (síndrome de Sturge-Weber) ${ }^{(2)}$. Muito raramente a forma circunscrita é observada em pacientes com a apresentação sistêmica da síndrome de Sturge-Weber ${ }^{(2)}$.

A primeira descrição do hemangioma da coróide foi feita por Leber ${ }^{(3)} \mathrm{em}$ 1868 e a descrição da sua aparência fundoscópica foi feita por $\mathrm{Fehr}^{(4)} \mathrm{em}$ 1905. A incidência exata é desconhecida. Cerca de 50\% dos tumores são difusos $^{(5-6)}$ mas é possível que os tumores circunscritos sejam subdiagnosticados devido à sua apresentação insidiosa ${ }^{(7)}$. A grande maioria dos casos acomete a raça branca ${ }^{(8-9)}$ sem predileção por sexo ${ }^{(10-11)}$. O tumor é congêni- 
to $^{(12)}$ mas geralmente o diagnóstico ocorre após o aparecimento de sintomas secundários, entre os 25 e 50 anos de idade ${ }^{(12)}$.

Vários exames diagnósticos como o ultra-som ${ }^{(13)}$, a angiofluresceinografia $^{(14)}$ e a ressonância magnética ${ }^{(15)}$ são utilizados na diferenciação do hemangioma da coróide com outros tumores, como o melanoma amelanocítico e o tumor metastático. O tratamento é indicado nas lesões sintomáticas e naquelas que apresentam risco de acometimento visual ${ }^{(16)}$.

A associação do hemangioma de coróide ao descolamento de retina representa uma causa importante de diminuição da acuidade visual e um desafio terapêutico. Os tratamentos já consagrados, como o laser de $\operatorname{argônio}^{(17)}$ e a betaterapia ${ }^{(18)}$, tornam-se muitas vezes ineficazes devido à dificuldade de acesso ao hemangioma. Desta forma, outras terapias são sugeridas, entre elas a termoterapia transpupilar ${ }^{(19-21)}$, a terapia fotodinâmica ${ }^{(22-25)}$ e a vitrectomia posterior com endolaser ${ }^{(26)}$, sendo a última motivo do presente estudo.

\section{RELATO DO CASO}

Paciente S. A. G., 41 anos, sexo feminino, branca, natural e procedente de Santos - SP. Apresentava mancha escura na visão do olho direito há uma semana. Relatava progressão da mancha para o campo inferior e diminuição súbita da acuidade visual há dois dias. Negava dor ou hiperemia ocular. Negava antecedentes pessoais ou familiares.

Ao exame oftalmológico, apresentava acuidade visual menor que 20/400 no olho direito (OD) e 20/20 no olho esquerdo (OE). A pressão intra-ocular era $10 \mathrm{mmHg}$ no OD e $13 \mathrm{mmHg}$ no OE. A biomicroscopia não mostrava alterações. Os reflexos pupilares estavam presentes. À fundoscopia do OD, observava-se elevação da retina neurossensorial nos quatro quadrantes e uma lesão sub-retiniana avermelhada no pólo posterior, com bordas pouco nítidas (Figura 1). O exame do OE era normal. O ultra-som do OD mostrava membranas móveis de alta refletividade na cavidade vítrea (retina) aderidas ao nervo óptico e presença de lesão sólida hiperecogênica, homogênea, no pólo posterior, com picos de média refletividade no seu interior, sugestiva de hemangioma de coróide (Figura 2). Foram solicitados exames para detecção de possível tumor primário: tomografia de crânio, tórax e abdomen, avaliação ginecológica, mamografia, CEA. Todos os exames foram normais. O diagnóstico presumido foi hemangioma de coróide com descolamento total de retina.

Dentre as opções de tratamento, optou-se pela vitrectomia posterior com endodrenagem, retinopexia, endofotocoagulação do tumor e colocação de gás $\mathrm{C}_{3} \mathrm{~F}_{8}$. A retina manteve-se aplicada nos quinze primeiros dias da cirurgia, quando então apresentou novo descolamento inferior da retina sobre o tumor (Figura 3). Optou-se por nova vitrectomia posterior com endofotocoagulação e colocação de óleo de silicone.

A paciente encontra-se no $6^{\circ}$ mês de pós-operatório com a retina aplicada, óleo de silicone na cavidade vítrea, hemangioma com marcas de fotocoagulação (inativo?), acuidade visual igual a 20/400 no OD (Figura 4).

\section{DISCUSSÃO}

O objetivo do tratamento do hemangioma da coróide é a resolução do descolamento seroso da retina no intuito de melhorar ou estabilizar a acuidade visual do paciente ${ }^{(27-28)}$.

Entre os tratamentos propostos, a fotocoagulação é o mais difundido $(44 \%)$, seguido pela betaterapia (4\%), radioterapia externa $(1 \%)$ e terapia cirúrgica $(1 \%)^{(1)}$.

A fotocoagulação com xenônio mostra-se eficaz na diminuição do crescimento do tumor e reabsorção do descolamento $^{(29)}$, porém a persistência dos descolamentos leva a baixa acuidade visual final ${ }^{(9)}$. O laser de argônio resulta na reabsorção do descolamento em $82,4 \%$ dos $\operatorname{casos}^{(17)}$; mas estudos

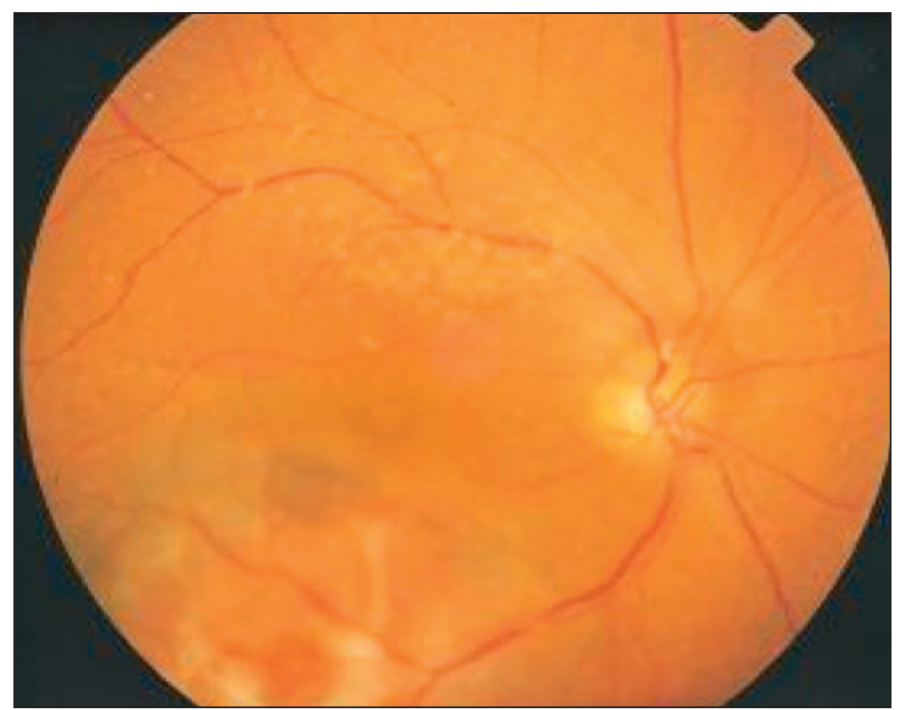

Figura 1 - Lesão sub-retiniana avermelhada no pólo posterior, com bordas pouco nítidas, e descolamento da retina neurossensorial nos quatro quadrantes

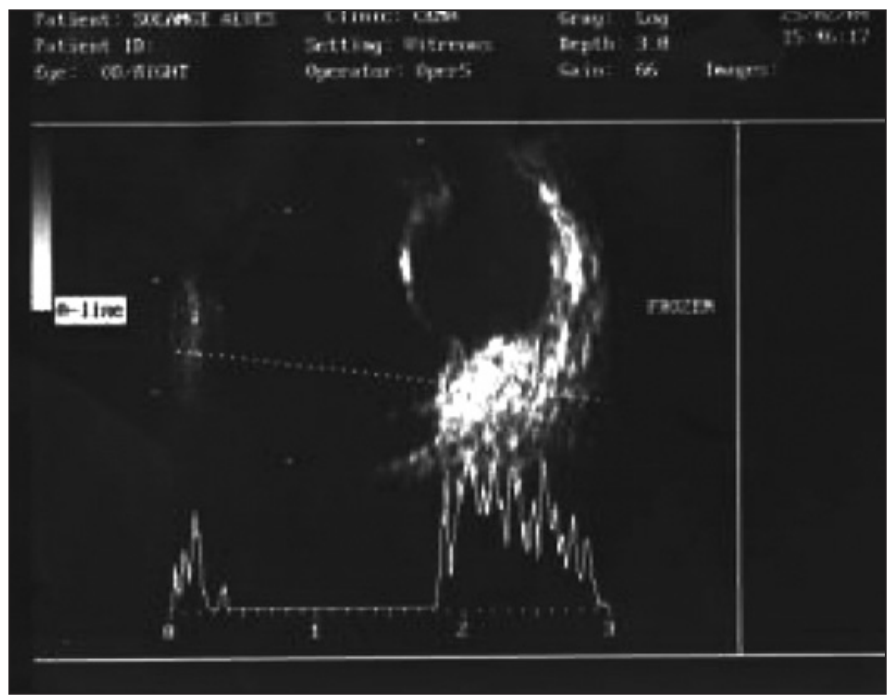

Figura 2 - Ultra-som ocular: lesão sólida hiperecogênica, homogênea, no pólo posterior, com picos de média refletividade no seu interior, sugestiva de hemangioma de coróide 


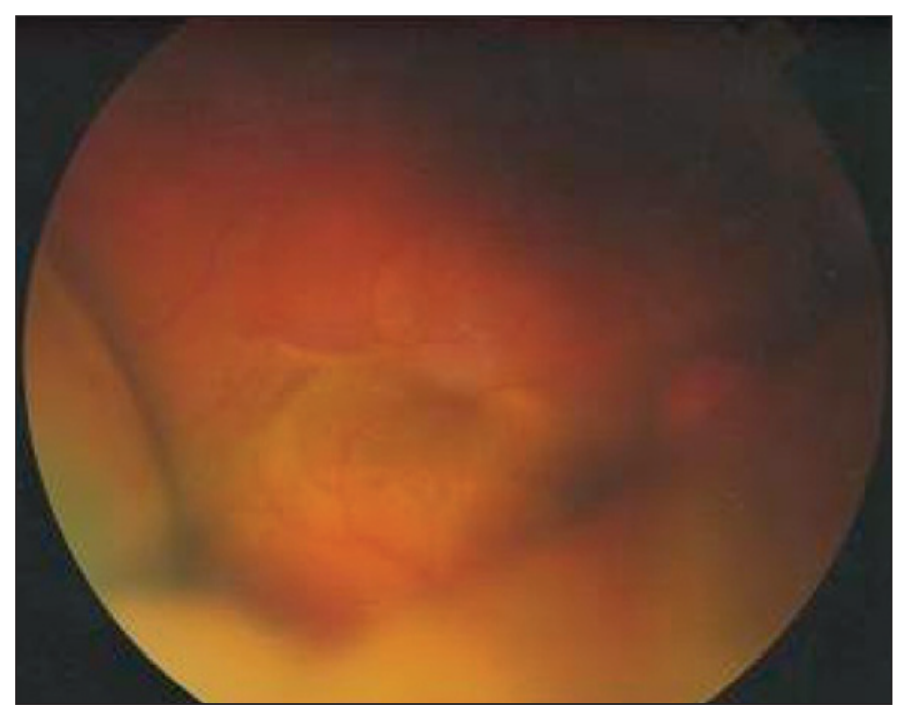

Figura 3 - Recidiva do descolamento inferior da retina sobre o tumor no $15^{\circ}$ pós-operatório

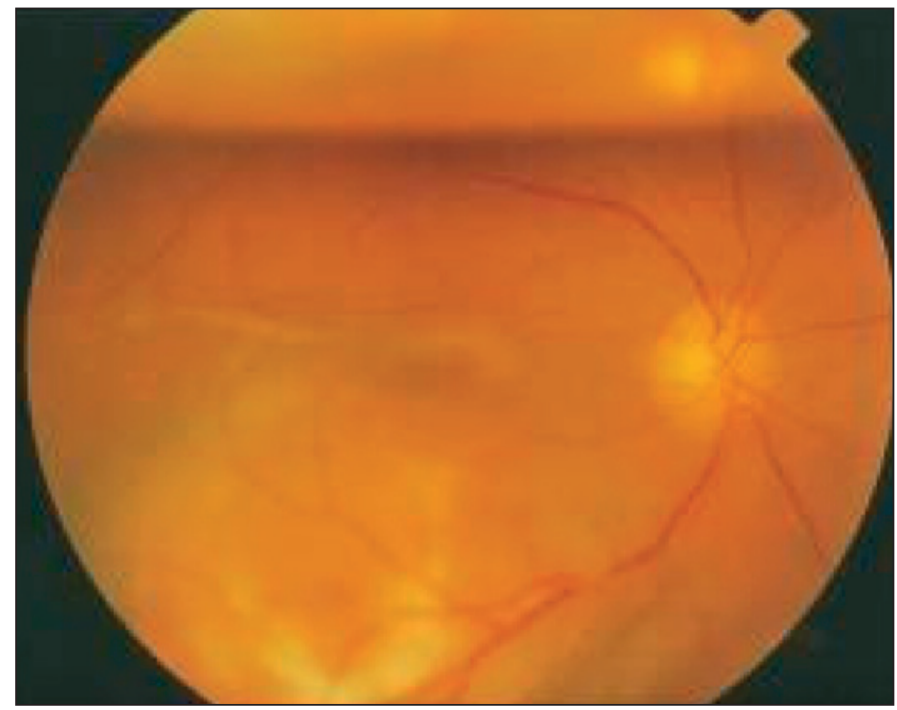

Figura 4 - 6ำ mês de pós-operatório: retina aplicada, óleo de silicone na cavidade vítrea, hemangioma com marcas de fotocoagulação

mostram altos índices de recorrência $(23,5 \%)^{(17,21)}$. São descritas associações entre fotocoagulação com argônio e acetazolamida sistêmica capazes de acelerar a absorção do descolamento $^{(30)}$. Complicações da própria fotocoagulação, como a síndrome de efusão uveal, foram descritas após o tratamento de hemangiomas difusos ${ }^{(31)}$. Outras complicações, como baixa acuidade visual final, foram relacionadas ao acometimento foveal pelo tumor ${ }^{(8)}$.

A crioterapia também é capaz de destruir o tumor e levar à reabsorção do líquido e conseqüente melhora da acuidade visual $^{(32)}$. Porém, sua utilização é restrita devido à dificuldade de acesso ao tumor.

A radioterapia externa, em doses fracionadas (2 Gy/dose), num total de $20 \mathrm{~Gy}$, causa a reabsorção do descolamento na maioria dos casos, mas não a destruição total do tumor ${ }^{(33-34)}$, uma vez que a arquitetura cavernosa destes tumores oferece mais resistência à radioterapi ${ }^{(33)}$. A principal causa de baixa visual a longo prazo é o desenvolvimento de fibrose subretiniana ${ }^{(33)}$.

A betaterapia com placa de paládio 103 (dose total igual a $2.900 \mathrm{cGy}$ ) pode levar à reabsorção do líquido sub-retiniano em quase todos os casos, com melhora inicial da acuidade visual em $60 \%$ dos pacientes ${ }^{(18)}$. Após 2 anos, observa-se piora da acuidade visual nos pacientes que desenvolvem maculopatia por irradiação(18). A betaterapia com placa de cobalto 60 obtém resultados semelhantes ${ }^{(34)}$.

A termoterapia transpupilar tornou-se uma técnica bastante utilizada nas lesões que envolvem a mácula, baseada no princípio de que o laser de diodo é absorvido mais profundamente pela coróide, poupando o epitélio pigmentar ${ }^{(35)}$. Os resultados iniciais mostram reabsorção do líquido na maioria dos $\operatorname{casos}^{(19)}$.

A terapia fotodinâmica possibilita o tratamento da área específica do tumor, poupando a retina e os vasos adjacentes, através da utilização de um contraste sensibilizante, a verteporfirina e do laser de diodo. Em trabalhos recentes, a reabsorção do líquido ocorreu em $94,8 \%{ }^{(36)}$ a $100 \%$ dos casos $^{(22-23)}$ com melhora da acuidade visual em $73 \%$ a $80 \%$ dos pacientes ${ }^{(22-23)}$.

A cirurgia vitreorretiniana é reservada aos casos de descolamento extenso da retina, nos quais outras opções terapêuticas tornam-se inviáveis pela dificuldade de acesso ao tumor. Alguns autores relataram um caso tratado com vitrectomia posterior e endofotocoagulação do tumor e colocação de óleo de silicone $^{(26)}$. O óleo de silicone foi removido após quatro semanas e a exsudação recidivou na oitava semana. Foi realizada termoterapia transpupilar na lesão, que regrediu completamente. No presente caso, optou-se pela vitrectomia posterior com endodrenagem, retinopexia, endofotocoagulação do tumor e colocação de gás $\mathrm{C}_{3} \mathrm{~F}_{8}$. A recidiva da exsudação, com descolamento da retina inferior, foi tratada com nova intervenção cirúrgica, endofotocoagulação e colocação de óleo de silicone, com resultado anatômico final satisfatório e estabilização da acuidade visual.

A acuidade visual final, numa revisão de Mashayekhi e Shields ${ }^{(36)}$, foi influenciada por três fatores: tempo de intervalo entre o aparecimento dos sintomas e o tratamento, visão inicial baixa e alterações crônicas da retina e epitélio pigmentar. A vitrectomia posterior surge como uma opção no tratamento do hemangioma da coróide associado a descolamentos extensos da retina, visando restabelecer a anatomia e diminuir as complicações funcionais tardias na retina.

\section{ABSTRACT}

To describe a case of circumscribed choroidal hemangioma with extensive retinal detachment treated with vitrectomy and endolaser photocoagulation. Interventional case report. A 41-year-old female patient was examined with a 7-day history 
of blurred vision and progression of visual loss in the right eye in the last 2 days. Ophthalmologic examination showed $20 / 400$ visual acuity in the right eye and an extensive retinal detachment with an elevated red lesion on the posterior pole. Ocular ultrasound showed high a reflective membranes in the vitreous cavity (retinal detachment) and an homogeneous hiperecogenic solid lesion suggestive of choroidal hemangioma. Systemic investigation showed no abnormalities. Posterior pars plana vitrectomy with endophotocoagulation and injection of $\mathrm{C}_{3} \mathrm{~F}_{8}$ gas was performed. After 15 days, an inferior retinal detachment was observed and the patient was submitted to a second vitrectomy with endophotocoagulation and silicon oil implant. After six months, the retina remains attached and the hemangioma shows no signs of exudation. Visual acuity remains 20/400. Posterior vitrectomy appears as an option for the treatment of circumscribed choroidal hemangiomas with extensive retinal detachment.

Keywords: Hemangioma; Choroid neoplasms; Vitrectomy; Retinal detachment; Case reports [publication type]

\section{REFERÊNCIAS}

1. Shields CL, Honavar SG, Shields JA, Cater J, Demirci H. Circumscribed choroidal hemangioma: clinical manifestations and factors predictive of visual outcome in 200 consecutive cases. Ophthalmology. 2001;108(12):2237-48. Erratum in: Ophthalmology. 2002;109(2):222.

2. Scott IU, Alexandrakis G, Cordahi GJ, Murray TG. Diffuse and circumscribed choroidal hemangiomas in a patient with Sturge-Weber syndrome. Arch Ophthalmol. 1999;117(3):406-7.

3. Leber T. Fall von cavernösem Sarcom der Aderhaut. Arch Ophthalmol. 1868; 14:221-7.

4. Fehr I. Ueber das Angiom der Aderhaut. Centralbl Prakt Augenheilk. 1905; 29:161-71.

5. Reese AB. Tumors of the eye. 3 ed. Hagerstown, MD: Harper \& Row; 1976. p. $277-82$.

6. Rosen E. Hemangioma of the choroid. Ophthalmologica. 1950;120(3):127-49.

7. Chisholm IH, Blach RK. Choroidal haemangioma, a diagnostic and therapeutic problem. Trans Ophthalmol Soc U K. 1973;93(0):161-9.

8. Anand R, Augsburger JJ, Shields JA. Circumscribed choroidal hemangiomas, Arch Ophthalmol. 1989;107(9):1338-42.

9. Augsburger JJ, Shields JA, Moffat KP. Circumscribed choroidal hemangiomas: long-term visual prognosis. Retina. 1981;1(1):56-61.

10. Gass JD. Differential diagnosis of intraocular tumors. A stereoscopic presentation. St Louis: Mosby-Year; 1974. p.114-29.

11. Witschel H, Font RL. Hemangioma of the choroid. A clinicopathologic study of 71 cases and a review of the literature. Surv Ophthalmol. 1976;20(6):415-31.

12. Heimann K. The development of the choroid in man. Choroidal vascular system. Ophthalmic Res. 1972;3:257-73.

13. Goes F, Benozzi J. Ultrasonographic aid in the diagnosis of choroidal hemangioma. Bull Soc Belge Ophtalmol. 1980;191:97-111.

14. Gass JD. Fluorescein angiography. An aid in the differential diagnosis of intraocular tumors. Int Ophthalmol Clin. 1972;12(1):85-120.

15. Shields JA, Shields CL. Intraocular tumor. In: Shields JA, Shields CL. Atlas of eyelid and conjunctival tumores. Philadelphia, WB Saunders; 1992. p.240-52.
16. Sanborn GE, Augsburger JJ, Shields JA. Treatment of circumscribed choroidal hemangiomas. Ophthalmology. 1982;89(12):1374-80.

17. Duquesne N, Bouchard O, Jean-Louis B, Bievelez B, Grange JD. [Argon laser photocoagulation of circumscribed choroidal hemangiomas] J Fr Ophtalmol. 2002;25(1):42-7. Erratum in: J Fr Ophtalmol. 2002;25(3):following table of contents. French.

18. Aizman A, Finger PT, Shabto U, Szechter A, Berson A. Palladium 103 (103Pd) plaque radiation therapy for circumscribed choroidal hemangioma with retinal detachment. Arch Ophthalmol. 2004;122(11):1652-6.

19. Rosa AAM, Roizemblat J. Termoterapia transpupilar como opção terapêutica para hemangioma circunscrito de coróide: relato de caso. Arq Bras Oftalmol. 2002;65(2):257-60.

20. Garcia-Arumi J, Ramsay LS, Guraya BC. Transpupillary thermotherapy for circumscribed choroidal hemangiomas. Ophthalmology. 2000;107(2):351-6 discussion 357.

21. Fuchs AV, Mueller AJ, Grueterich M, Ulbig MW. Transpupillary thermotherapy (TTT) in circumscribed choroidal hemangioma. Graefes Arch Clin Exp Ophthalmol. 2002;240(1):7-11.

22. Scott IU, Gorscak J, Gass JD, Feuer WJ, Murray TG. Anatomic and visual acuity outcomes following thermal laser photocoagulation or photodynamic therapy for symptomatic circumscribed choroidal hemangioma with associated serous retinal detachment. Ophthalmic Surg Lasers Imaging. 2004;35(4):281-91.

23. Verbraak FD, Schlingemann RO, Keunen JE, de Smet MD. Longstanding symptomatic choroidal hemangioma managed with limited PDT as initial or salvage therapy. Graefes Arch Clin Exp Ophthalmol. 2003;241(11):891-8.

24. Jurklies B, Anastassiou G, Ortmans S, Schuler A, Schilling H, SchmidtErfurth U, et al. Photodynamic therapy using verteporfin in circumscribed choroidal haemangioma. Br J Ophthalmol. 2003;87(1):84-9.

25. Singh AD, Kaiser PK, Sears JE, Gupta M, Rundle PA, Rennie IG. Photodynamic therapy of circumscribed choroidal haemangioma. $\mathrm{Br} \mathrm{J}$ Ophthalmol. 2004;88(11):1414-8.

26. Hirakata A, Okada AA, Asakawa M, Mitsui K, Hida T. [A case of choroidal hemangioma with bullous exudative retinal detachment treated successfully by transpupillary thermotherapy] Nippon Ganka Gakkai Zasshi. 2001;105(6):41520. Japanese.

27. Gass JDM. Stereoscopic atlas of macular diseases; diagnosis and treatment. St Louis: CV Mosby; 1987.

28. Shields JA, Shields CL, Materin MA, Marr BP, Demirci H, Mashayekhi A. Changing concepts in management of circumscribed choroidal hemangioma: the 2003 J. Howard Stokes Lecture, Part 1. Ophthalmic Surg Lasers Imaging. 2004;35(5):383-94.

29. Mackensen D, Meyer-Schwickerath G. [Diagnosis and treatment of choroidal haemangioma (author's transl)] Klin Monatsbl Augenheilkd. 1980;177(1):1623. German.

30. Althaus C, Sundmacher R. [Combination therapy of circumscribed choroid hemangioma in contralateral Sturge-Weber syndrome with acetazolamide and laser coagulation] Klin Monatsbl Augenheilkd. 1996;208(4):239-42. German.

31. Ribstein G, Turut P, Madelain J, Malka I. [Uveal effusion syndrome after argon laser treatment in a case of Sturge Weber Krabbe syndrome] Bull Soc Ophtalmol Fr. 1990;90(2):189-92. French.

32. Humphrey WT. Choroidal hemangioma: Response to cryotherapy. Ann Ophthalmol. 1979;11(1):100-4.

33. Schilling H, Sauerwein W, Lommatzsch A, Friedrichs W, Brylak S, Bornfeld N, et al. Long-term results after low dose ocular irradiation for choroidal haemangiomas. Br J Ophthalmol. 1997;81(4):267-73. Comment in: Br J Ophthalmol. 1997;81(4):258-9.

34. Eide N, Syrdalen P, Tausjo J, Tvera K. External radiotherapy for circumscribed choroidal haemangiomas using a modified retinoblastoma technique. Acta Ophthalmol Scand. 1995;73(6):547-50.

35. Lanzetta P, Virgili G, Ferrari E, Menchini U. Diode laser photocoagulation of choroidal hemangioma. Int Ophthalmol. 1995-96;19(4):239-47.

36. Mashayekhi A, Shields CL. Circumscribed choroidal hemangioma. Curr Opin Ophthalmol. 2003;14(3):142-9. 